\title{
STRONG MEASURE ZERO SETS IN POLISH GROUPS
}

\author{
MICHAEL HRUŠÁK AND JINDŘICH ZAPLETAL
}

\begin{abstract}
In the context of arbitrary Polish groups, we investigate the Galvin-Mycielski-Solovay characterization of strong measure zero sets as those sets for which a meager collection of right translates cannot cover the whole group.
\end{abstract}

\section{Introduction}

Emile Borel defined the collection of strong measure zero sets of reals:

Definition 1.1. A set $A \subset \mathbb{R}$ is a strong measure zero set if for every sequence $\left\langle\varepsilon_{n}: n \in \omega\right\rangle$ of positive real numbers there are intervals $I_{n} \subset \mathbb{R}$ of respective legths $\varepsilon_{n}$ such that $A \subset \bigcup_{n} I_{n}$.

It is clear that every countable set of reals has strong measure zero. The failure of the effort to produce an uncountable strong measure zero set led Borel to the following conjecture:

Conjecture 1.2 (Borel conjecture). The strong measure zero sets are exactly the countable sets.

Today we know [8] that under the Continuum Hypothesis there are uncountable strong measure zero sets, and in a certain model of ZFC (the Laver model) there are no uncountable strong measure zero sets. Thus, the Borel conjecture is not decidable in ZFC set theory. One interesting feature of strong measure zero sets is the following characterization, proved by Galvin, Mycielski, and Solovay:

FACT $1.3([3])$. A set $A \subset \mathbb{R}$ is strong measure zero if and only if for every meager set $M \subset \mathbb{R}, A+M \neq \mathbb{R}$.

Received September 15, 2016; received in final form May 6, 2017.

The first author was supported by a PAPIIT grant IN108014 and CONACyT Grant 177758. The second author was supported by NSF Grant DMS 1161078.

2010 Mathematics Subject Classification. 03E35, 22A05, $20 \mathrm{~B} 35$. 
The topic of this paper is a generalization of strong measure zero sets to arbitrary Polish groups and the verification of the above characterization in this more general context. One should remark right away that if Borel conjecture holds, then all strong measure zero sets in any given Polish group are countable and the GMS characterization holds automatically. Thus, one is interested only in the context of the Continuum Hypothesis, which is in a sense opposite of that of Borel conjecture.

Definition $1.4(Z F C+C H)$. A Polish group $G$ is GMS if for every set $A \subset G, A$ is strong measure zero if and only if for every meager set $M \subset G$, $A \cdot M \neq G$.

The choice of the Continuum Hypothesis context is critical also because of the syntactical complexity of the GMS property. The statement "G is GMS" is $\Pi_{1}^{2}(G)$, and as such is decided in the $\Omega$-logic by the Continuum Hypothesis. Also, if the decision is positive under $\mathrm{CH}$, it is positive already in $\mathrm{ZFC}$ [7]; in other words, under $\mathrm{CH}$ there are as many non-GMS groups as possible.

An obvious question is whether there is a restatement of the above definition which would make an equivalent sense even in the context of ZFC. There is a natural conjecture which, if true, would identify the class of GMS groups with a classical class of groups:

Conjecture $1.5(\mathrm{ZFC}+\mathrm{CH})$. A Polish group $G$ is GMS if and only if it is locally compact.

The right-to-left implication has been known [2, Section 534], [6], [4]. We investigate the problematic left-to-right implication and confirm it in two extensive classes of groups.

THEOREM 1.6. The conjecture holds in the class of groups with bi-invariant metric, and in the class of closed subgroups of $S_{\infty}$.

This greatly extends the result of [4], where it is proved that the BaerSpecker group $\mathbb{Z}^{\omega}$ is not GMS.

We use the set theoretic notational standard of [5]. Let $G$ be a group. Unless $G=\mathbb{R}$, we denote the group operation by $\cdot$. If $g \in G$ and $A \subset G$, then $g A$ denotes the set $\{g h: h \in A\}$; similar usage prevails for right translates and inverses of subsets of $G$.

\section{Strong measure zero sets in arbitrary Polish groups}

In this section, we define the strong measure zero sets in arbitrary Polish groups and verify their basic properties.

Definition 2.1. Let $G$ be a Polish group. A set $A \subset G$ is left strong measure zero, lsmz if for every sequence $\left\langle U_{n}: n \in \omega\right\rangle$ of nonempty open subsets of the group there are elements $g_{n} \in G$ for $n \in \omega$ such that $A \subset \bigcup_{n} g_{n} U_{n}$. 
Theorem 2.2. Let $G$ be a Polish group.

(1) The left strong measure zero sets form a bi-invariant $\sigma$-ideal;

(2) if the Borel conjecture holds, then the left strong measure zero sets are exactly the countable sets.

Proof. For (1), it is easy to verify that the left strong measure zero sets form a $\sigma$-ideal. Suppose $A=\bigcup_{n} A_{n}$ and for every $n \in \omega$ the set $A_{n} \subset G$ is lsmz. To verify that $A$ is $\mathrm{lsmz}$, suppose that $U_{m}$ for $m \in \omega$ are nonempty open subsets of the group. Let $\left\{b_{n}: n \in \omega\right\}$ be a partition of $\omega$ into countably many infinite sets, and use the lsmz property of each set $A_{n}$ to find group elements $g_{m}$ for $m \in \omega$ such that for every $n \in \omega, A_{n} \subset \bigcup_{m \in b_{n}} g_{m} U_{m}$. Then $A \subset \bigcup_{m \in \omega} g_{m} U_{m}$.

To verify the invariance, suppose that $A \subset G$ is a left strong measure zero set and $g \in G$ is an arbitrary element. To see that $g A$ is lsmz, suppose that $\left\langle U_{n}: n \in \omega\right\rangle$ is a sequence of nonempty open subsets of the group. As $A$ is lsmz, there are elements $g_{n} \in G$ such that $A \subset \bigcup_{n} g_{n} U_{n}$, and then $g A \subset \bigcup_{n} g g_{n} U_{n}$. This confirms that the set $g A$ is lsmz. To verify that the set $A g$ is lsmz, suppose that $\left\langle U_{n}: n \in \omega\right\rangle$ is a sequence of nonempty open subsets of the group. As $A$ is lsmz, there are elements $g_{n} \in G$ such that $A \subset \bigcup_{n} g_{n} U_{n} g^{-1}$. Then, $A g \subset \bigcup_{n} g_{n} U_{n}$, and so the set $A g$ is lsmz as required.

For (2), recall the result of Carlson: the Borel conjecture implies that separable strong measure zero metric spaces must be countable. See $[1$, Theorem 3.2].

The following two theorems record what is known regarding the GMS characterization of left strong measure zero sets in general Polish groups.

Theorem 2.3. Let $G$ be a Polish group.

(1) Whenever $A \subset G$ is a set and $A \cdot C \neq G$ for every nowhere dense set $C \subset G$, then $A$ is lsmz;

(2) if $G$ is locally compact and $A \subset G$ is a lsmz set, then $A \cdot C \neq G$ for every meager set $C \subset G$.

Proof. For (1), fix a set $A \subset G$. Let $\left\langle U_{n}: n \in \omega\right\rangle$ be a sequence of nonempty open subsets of $G$. Let $\left\langle g_{n}: n \in \omega\right\rangle$ be a sequence of elements of the group such that the set $\bigcup_{n} g_{n} U_{n} \subset G$ is open dense. Let $C=\left(G \backslash \bigcup_{n} g_{n} U_{n}\right)^{-1}$; as the inverse map is a self-homeomorphism of $G$, the set $C \subset G$ is closed nowhere dense. Now, since $A \cdot C \neq G$, there is a group element $g \in G$ such that $g \notin A \cdot C$. This means that for every $h \in A, g \notin h \cdot C$, so $h \notin g \cdot C^{-1}$, in other words $h \in \bigcup_{n} g \cdot g_{n} U_{n}$. Thus, $A \subset \bigcup_{n} g \cdot g_{n} U_{n}$ and the set $A \subset G$ is lsmz.

For (2), see [6] or [2, Section 534] or [4]. 
The attempts to prove the converse to Theorem 2.3(1) for Polish groups which are not locally compact are the starting point of the current paper. To violate the converse, we introduce a special type of nowhere dense set.

Definition 2.4 ([4]). Let $G$ be a Polish group. A nonempty set $C \subset G$ is anti-GMS if it is nowhere dense and for every sequence $\left\langle U_{n}: n \in \omega\right\rangle$ of open neighborhoods of 1 there is a sequence $\left\langle g_{n}: n \in \omega\right\rangle$ of elements of $G$ such that for every $g \in G$, the set $g \cdot \bigcup_{n} g_{n} \cdot U_{n}$ is dense in $C$.

Theorem 2.5 $(\mathrm{ZFC}+\mathrm{CH})$. Let $G$ be a Polish group. If there is an anti$G M S$ set in $G$, then the group $G$ is not GMS.

Proof. Let $C \subset G$ be an anti-GMS set; passing to a closure if necessary, we may assume that it is closed. We will construct a lsmz set $A \subset G$ such that $A \cdot C^{-1}=G$.

Let $\left\{U_{n}^{\alpha}: n \in \omega, \alpha \in \omega_{1}\right\}$ be an enumeration of all $\omega$-sequences of nonempty basic open subsets of $G$. Since $C$ is an anti-GMS set, we can choose elements $\left\{g_{n}^{\alpha}: n \in \omega, \alpha \in \omega_{1}\right\}$ such that for every $\alpha \in \omega_{1}$ the set $\bigcup_{n} g_{n}^{\alpha} U_{n}^{\alpha}$ is dense in $C$. Let $\left\{h_{\alpha}: \alpha \in \omega_{1}\right\}$ be an enumeration of the group $G$. For each ordinal $\alpha \in \omega_{1}$ we will find elements $g_{\alpha} \in G$ and $k_{\alpha} \in C$ so that

- if $\beta<\alpha$, then $g_{\alpha} \in \bigcup_{n} g_{n}^{\beta} U_{n}^{\beta}$;

- $g_{\alpha} \cdot k_{\alpha}^{-1}=h_{\alpha}$.

Let $A=\left\{g_{\alpha}: \alpha \in \omega_{1}\right\}$. The first item ensures that $A$ is a lsmz set. The second item shows that $G=A \cdot C^{-1}$, and this will complete the proof of the theorem.

To construct $g_{\alpha}, k_{\alpha}$, note that for every $\beta \in \alpha$, the sets $\bigcup_{n} h_{\alpha}^{-1} g_{n}^{\beta} U_{n}^{\beta}$ are open dense in $C$. Since there are only countably many such sets, there is some $k_{\alpha} \in C$ which belongs to all of them. Let $g_{\alpha}=h_{\alpha} \cdot k_{\alpha}$. By the choice of $k_{\alpha}$, for every $\beta \in \alpha$ it is the case that $g_{\alpha} \in \bigcap_{\beta \in \alpha} \bigcup_{n} g_{n}^{\beta} U_{n}^{\beta}$ as desired. Finally, $g_{\alpha} k_{\alpha}^{-1}=h_{\alpha} k_{\alpha} k_{\alpha}^{-1}=h_{\alpha}$ as desired in the second item and the proof is complete.

We do not know if the converse to Theorem 2.5 holds. Nevertheless, the failure of GMS property in Polish groups in this paper is always obtained through a construction of an anti-GMS set. Note that if a set $C \subset G$ is antiGMS then its closure is anti-GMS as well. Thus, the existence of anti-GMS set is a projective statement.

As a final remark in this section, in Definition 2.1 one multiplies by the group elements from the left. It is also possible to consider a similar definition with multiplication from the right: a set $A \subset G$ is right strong measure zero, $r s m z$ if for every sequence $\left\langle U_{n}: n \in \omega\right\rangle$ of nonempty open subsets of the group there are elements $g_{n} \in G$ for $n \in \omega$ such that $A \subset \bigcup_{n} U_{n} g_{n}$. We will not work on the difference between the left and right strong measure zero sets beyond the following basic theorem. 
Theorem 2.6. Let $G$ be a Polish group.

(1) For every set $A \subset G, A$ is lsmz if and only if $A^{-1} \subset G$ is rsmz;

(2) if $G$ admits a bi-invariant metric or the Borel conjecture holds then the lsmz and rsmz sets coincide;

(3) if $G=S_{\infty}$ and the continuum hypothesis holds, then the ideals of lsmz and rsmz sets are not the same.

Proof. For the left-to-right direction of (1), suppose that $A$ is lsmz. To verify the rsmz of $A^{-1}$, suppose that $\left\langle U_{n}: n \in \omega\right\rangle$ is a sequence of nonempty open subsets of the group. By the lsmz property of the set $A$, there is a sequence $\left\langle g_{n}: n \in \omega\right\rangle$ of elements of the groups such that $A \subset \bigcup_{n} g_{n} U_{n}^{-1}$. Then, $A^{-1} \subset \bigcup_{n} U_{n} g_{n}^{-1}$, verifying the rsmz of $A^{-1}$. The right-to-left implication is similar.

For (2), if $d$ is a bi-invariant metric on the grup $G$, then both left and right shifts of $d$-balls of a fixed radius $\varepsilon>0$ are exactly the $d$-balls of radius $\varepsilon$. In the definition of both lsmz and rsmz sets, shrinking the sets $U_{n}$ if necessary we may assume that they are $d$-balls, and then the definitions of rsmz and lsmz coincide. If the Borel conjecture holds, then both the lsmz and rsmz ideal coincide with the $\sigma$-ideal of countable sets.

For (3), in view of (1) it is enough to find a set $A \subset S_{\infty}$ such that $A$ is left strong measure zero while $A^{-1}$ is not. Let $\left\langle x_{\alpha}: \alpha \in \omega_{1}\right\rangle$ be an enumeration of increasing functions in $\omega^{\omega}$ and let $\left\langle y_{\alpha}: \alpha \in \omega_{1}\right\rangle$ be an enumeration of $S_{\infty}^{\omega}$. By transfinite induction on $\alpha$ we will produce points $z_{\alpha} \in S_{\infty}^{\omega}$ and $g_{\alpha} \in S_{\infty}$ such that

(1) for every $\beta \in \alpha, g_{\alpha} \in \bigcup_{n}\left[z_{\beta}(n)\left\lceil x_{\beta}(n)\right]\right.$ and $g_{\alpha}^{-1} \notin \bigcup_{n}\left[y_{\alpha}^{-1}(n)\lceil n]\right.$.

Once this is done, it is clear that the set $A=\left\{g_{\alpha}: g \in \omega_{1}\right\}$ is strong left measure zero while the set $A^{-1}$ is not. An additional inductive hypothesis on the points $z_{\alpha}$ will be the following:

(2) Let $s$ be a finite injection from $\omega$ to $\omega$. Let $m<k$ be the first two numbers not in the range of $s$. We require that there will be a set $a \subset \omega$ of size at least $k+1$ such that the finite injections $z_{\alpha}(n)\left\lceil x_{\alpha}(n)\right.$ for $n \in a$ extend $s$, they all have $m$ in their range, neither has $k$ in their range, and their preimages of $m$ are pairwise distinct.

Now, suppose that $z_{\beta}, x_{\beta}$ have been found for $\beta \in \alpha$. It is easy to find $z_{\alpha}$ satisfying (2); this does not use the inductive assumption at all. To construct the point $g_{\alpha}$, choose an enumeration $\left\{\beta_{i}: i \in \omega\right\}=\alpha$. By induction on $i \in \omega$ find $n_{i} \in \omega$ such that the finite injections $s_{i}=z_{\beta_{i}}\left(n_{i}\right)\left\lceil x_{\beta_{i}}\left(n_{i}\right)\right.$ form a chain with respect to inclusion, and writing $m_{i}$ for the smallest number not in the range of $s_{i}, m_{i} \in \operatorname{rng}\left(s_{i+1}\right)$ and $\left[s_{i}\right]^{-1} \cap \bigcup_{n \leq m_{i}}\left[y_{\alpha}^{-1}(n)\lceil n]=0\right.$. Once this is done, the point $x_{\alpha}=\bigcup_{i} s_{i}$ is as required.

To perform the induction on $i$, start with $s_{-1}=0$. Suppose $s_{i}$ has been constructed. Let $m<k$ be the first two numbers not in the range of $s_{i}$. Use 
the induction hypothesis (2) at $\beta_{i}$ and $s_{i}$ to find a number $n_{i} \in \omega$ such that the injection $s_{i+1}$ contains $m$ in its range, does not contain $k$ in its range, and for every $m<n<k$, the preimages of $m$ under $y_{\alpha}(n)$ and $s_{i+1}$ are distinct. Then, $s_{i+1}$ works as desired.

\section{Submeasures on groups}

A useful tool for packaging the proof of Theorem 1.6 in the case of groups with bi-invariant metric is the concept of left invariant submeasure on a topological group. Recall that a submeasure $\mu$ on a Polish space $X$ is outer regular if for every set $A \subset X, \mu(A)=\inf \{\mu(O): A \subset O, O$ open $\}$. The submeasure $\mu$ is non-atomic if $\mu(\{x\})=0$ for every point $x \in X$.

Theorem 3.1. Let $G$ be an uncountable Polish group. There is a left invariant, outer regular, countably subadditive, nonatomic, nonzero submeasure on $G$.

Proof. It will be enough to produce a local inclusion-decreasing basis $\left\langle U_{n}: n \in \omega\right\rangle$ at 1 such that whenever $m \in \omega$ is a number and $a_{n} \subset G$ are sets of respective size $n$ for all $n>m$, then $U_{m}$ is not a subset of $\bigcup_{n>m} a_{n} \cdot U_{n}$. Once this is done, define the submeasure $\mu$ by setting $\mu(A)=\inf \left\{\sum_{i} 1 / n_{i}:\left\langle g_{i}, n_{i}: i \in \omega\right\rangle\right.$ are such that $\left.A \subset \bigcup_{i} g_{i} U_{n_{i}}\right\}$. The requested properties of $\mu$ follow from the definition. The only important point is that $\mu\left(U_{n}\right)=1 / n$, in particular open sets have nonzero $\mu$-mass. This, however, follows from the initial properties of the local basis.

To construct the local basis, choose a left invariant metric $d$ and a complete metric $e$ for the group $G$. By induction find the open sets $U_{n} \subset G$ together with sets $b_{n} \subset U_{n}$ of size $n+1$ such that

- writing $\varepsilon_{n}$ for the $d$-diameter of $U_{n+1}$, the points in $b_{n}$ are $d-3 \varepsilon_{n}$ distant from each other and also from the complement of $U_{n}$;

- for every $m<n$ and every choice $g_{i} \in b_{i}$ for $m<i<n$ the sets $\prod_{m<i<n} g_{i}$. $U_{n}$ have $e$-diameter $<1 / n$.

To check the desired properties of the basis, suppose that $m \in \omega$ is a number and $a_{n} \subset G$ are sets of respective size $n$ for all $n>m$. By induction on $n>m$ choose elements $g_{n} \in b_{n}$ so that the set $\prod_{m<i<n} g_{i} U_{n}$ is disjoint from all sets $g U_{n}$ for $g \in a_{n}$. This is possible since the metric $d$ is left-invariant and so each set $g U_{n}$ for $g \in a_{n}$ intersects at most one of the sets $\prod_{m<i<n} g_{i} \cdot h \cdot U_{n}$ for $h \in b_{n}$. The closures of the sets $\prod_{m<i<n} g_{i} U_{n}$ for $n<m$ form an inclusiondecreasing sequence with $e$-diameter tending to zero and so their intersection is nonempty by the completeness of the metric $e$. The point in their intersection belongs to $U_{m}$ but not to the set $\bigcup_{n>m} a_{n} \cdot U_{n}$. This completes the proof.

It is entirely natural to consider left invariant submeasures in conjunction with left strong measure zero sets, as the following theorem, due to Jan 
Grebík, shows. Lsmz sets are in a sense exactly the universally left invariant submeasure zero sets.

TheOrem 3.2. Let $G$ be a Polish group, and $A \subset G$ be a set. The following are equivalent:

(1) A is lsmz;

(2) for every left invariant, outer regular, countably subadditive, nonatomic submeasure $\mu$ on $G, \mu(A)=0$.

Proof. To see why (1) implies (2), suppose that $A$ is lsmz, $\mu$ is a left invariant submeasure on $G$, and $\varepsilon>0$ is a real number. To show that $\mu(A)<$ $\varepsilon$, just use the assumption that $\mu$ is nonatomic to find open neighborhoods $U_{n} \subset G$ of the unit for $n \in \omega$ such that $\sum_{n} \mu\left(U_{n}\right)<\varepsilon$. Use the assumption that $A$ is lsmz to find group elements $g_{n} \in G$ for $n \in \omega$ such that $A \subset \bigcup_{n} g_{n} \cdot U_{n}$. Finally use the countable subadditivity and invariance of $\mu$ to conclude that $\mu(A) \leq \sum_{n} \mu\left(g_{n} \cdot U_{n}\right)<\varepsilon$ as desired.

To see why (2) implies (1), suppose that (2) is satisfied and $U_{n} \subset G$ for $n \in \omega$ are nonempty open sets; we must find group elements $g_{n} \in G$ for $n \in \omega$ such that $A \subset \bigcup_{n} g_{n} \cdot U_{n}$. Thinning out the sets $U_{n}$ and shifting them on the left, we may assume that they are all neighborhoods of the unit and form a basis at the unit. Find numbers $n_{i} \in \omega$ for $i \in \omega$ such that $n_{i+1}>2 n_{i}$, write $\mathcal{O}=\left\{A_{n_{i}}: i \in \omega\right\}$ and define a submeasure $\mu$ on the group $G$ by setting $w\left(A_{n_{0}}\right)=1$ and $w\left(A_{n_{i+1}}\right)=1 / n_{i}$ and $\mu(B)=\inf \left\{\sum_{j} w\left(V_{j}\right): V_{j} \in \mathcal{O}\right.$ and there are group elements $g_{j} \in G$ such that $\left.B \subset \bigcup_{j} g_{j} \cdot V_{j}\right\}$. It is immediate that this is a left invariant, countably subadditive etc. submeasure on $G$. By (2), $\mu(A)<1$ holds, and so there are sets $V_{j} \in \mathcal{O}$ and group elements $g_{j}$ such that $A \subset \bigcup_{j} g_{j} \cdot V_{j}$ and moreover, $\sum_{j} w\left(V_{j}\right)<1$. The latter inequality shows that for every $i \in \omega$, the set $U_{n_{i+1}}$ occurs fewer than $n_{i}$-many times among the $V_{j}$ 's, and the set $U_{n_{0}}$ does not occur at all. The indexes $n_{i+1}$ increase so fast that it is possible to find sets $W_{j} \in\left\{U_{n}: n \in \omega\right\}$ for $j \in \omega$ such that $W_{j} \supset V_{j}$ and each $U_{n}$ occurs at most once among the $W_{j}$ 's. But then, $A \subset \bigcup_{j} g_{j} \cdot W_{j}$ and the set $A$ is lsmz as desired.

\section{Groups with bi-invariant metric}

TheOREM 4.1 (ZFC+CH). Suppose that $G$ is a non-locally compact Polish group with a bi-invariant metric. Then $G$ is not GMS.

Proof. By Theorem 2.5 it is enough to construct an anti-GMS set in the group $G$. Let $\mu$ be a left invariant countably subadditive diffuse submeasure on $G$. We will build a closed nowhere dense set $C \subset G$ such that whenever $O \subset G$ is an open set with nonempty intersection with $C$, there are nonempty open sets $\left\{Q_{m}: m \in \omega\right\}$, all subsets of $O$, all left translates of each other, such that the numbers $\mu\left(Q_{m} \backslash C\right)$ tend to zero as $m$ tends to infinity. Such a set $C \subset G$ will be anti-GMS. 
To see this, choose a countable collection of open neighborhoods $\left\{U_{n}: n \in\right.$ $\omega\}$ of the unit; shrinking if necessary, we may assume that their diameters tend to zero. Choose a pairwise disjoint collection $\left\{a_{O}: O \subset G\right.$ basic open $\}$ of infinite subsets of $\omega$. For each $O$ such that $O \cap C \neq 0$ find an open neighborhood $W_{O} \subset G$ of the unit such that $O$ contains infinitely many left translates of $W_{O}$ such that the $\mu$-masses of their intersections with the complement of $C$ tend to 0 . Removing finitely many elements from $a_{O}$ if necessary, we may find an open neighborhood $V_{O}$ of the unit such that for each $n \in a_{O}$ $V_{O} \cdot U_{n} \subset W_{O}$. Now, pick elements $g_{n}, h_{n} \in G$ for $n \in a_{O}$ such that $h_{n} W_{O} \subset O$ and $\mu\left(h_{n} W_{O} \backslash C\right)<\mu\left(U_{n}\right)$, and $G=\bigcup_{n \in a_{O}} h_{n} V_{O} g_{n}^{-1}$. This is possible since there is a bi-invariant compatible metric. We claim that the group elements $\left\{g_{n}: n \in \bigcup_{O} a_{O}\right\}$ work as desired.

Indeed, if $g \in G$ is an arbitrary element and $O \subset G$ is a basic open set with nonempty intersection with $C$, there must be a number $n \in a_{O}$ such that $g \in h_{n} V_{O} g_{n}^{-1}$, or in other words $g \cdot g_{n} \in h_{n} V_{O}$. Then $g \cdot g_{n} U_{n} \subset h_{n} V_{O} U_{n} \subset$ $h_{n} W_{O} \subset O$. Now, $\mu\left(h_{n} W_{O} \backslash C\right)<\mu\left(U_{n}\right)$ and so $C \cap O \cap g \cdot g_{n} U_{n} \neq 0$ as desired.

The construction of the nowhere dense closed set $C \subset G$ is performed by a routine induction. Let $\left\langle O_{k}: k \in \omega\right\rangle$ be an enumeration of a basis for the group $G$. By induction on $k \in \omega$ build sets $P_{k} \subset G$ and countable sets $A_{k}$ such that

1. $P_{k}$ is an increasing sequence of open subsets of $G$ and $O_{k} \cap P_{k+1} \neq 0$;

2. $A_{k}$ is a countable set of pairs of the form $\langle Q, \varepsilon\rangle$ such that $Q \subset G$ is open, $\varepsilon>0$. No point of $G$ belongs to more than $k$ many sets mentioned in $A_{k}$;

3. whenever $\langle Q, \varepsilon\rangle \in A_{k}$ then $\mu\left(\bar{P}_{k+1} \cap Q \backslash \bar{P}_{k}\right)<2^{-k} \varepsilon$. Moreover, if $k$ is the smallest such that $\langle Q, \varepsilon\rangle \in A_{k}$ then $\bar{P}_{k} \cap Q=0$;

4. either (a) $O_{k} \subset P_{k+1}$, or (b) the set $A_{k+1}$ contains an infinite collection of open sets, all left translates of each other, all subsets of $O_{k}$, paired with real numbers tending to zero.

In the end, let $C=G \backslash \bigcup_{k} P_{k}$. By the first item, this is a closed nowhere dense set. By the third item, whenever $\langle Q, \varepsilon\rangle \in \bigcup_{k} A_{k}$ then $\mu(Q \backslash C)<\varepsilon$. Now, if $O_{k}$ is a basic open set with nonempty intersection with $C$, then (b) must have occurred at $k$ in the last item, and so the set $O_{k}$ contains an infinite collection of open subsets with the required properties.

To perform the induction, start with $P_{0}=A_{0}=0$. If $P_{k}$ has been constructed, the next step divides into two cases, corresponding to (a) and (b) of the last item. Either $O_{k} \subset \bar{P}_{k}$, in which case let $A_{k+1}=A_{k}$ and $P_{k+1}=P_{k} \cup O_{k}$, and proceed to $k+1$. If $O_{k} \not \subset \bar{P}_{k}$, find disjoint open sets $R, S \subset O_{k} \backslash \bar{P}_{k}$. Use the second item to find a nonempty open subset $R^{\prime} \subset R$ which is either disjoint from or a subset of every open set mentioned in $A_{k}$. Since the submeasure $\mu$ is diffuse, there is a nonempty open set $R^{\prime \prime} \subset R^{\prime}$ with $\mu\left(\bar{R}^{\prime \prime}\right)$ so small that $P_{k+1}=P_{k} \cup R^{\prime \prime}$ is going to satisfy the demands of the third item for all pairs $\langle Q, \varepsilon\rangle \in A_{k}$ with $R^{\prime} \subset Q$. Finally, use the fact that 
the set $S$ is not compact to find infinitely many balls of equal diameter with pairwise disjoint closures inside $S$, and include them all in $A_{k+1}$, paired with some real numbers tending to zero.

\section{Closed subgroups of $S_{\infty}$}

THEOREM 5.1 (ZFC+CH). Let $G$ be a non-locally-compact, closed subgroup of $S_{\infty}$. Then $G$ is not $G M S$.

Proof. By Theorem 2.5, it is enough to construct an anti-GMS set $C \subset G$. By the lack of local compactness, there is an infinite set $a \subset \omega$ such that for every $n \in a$ there are infinitely many $m$ such that there is $g \in G$ for which $g\lceil n$ is the identity and $g(n)=m$. For numbers $n<m$ both in $a$ define $R_{n, m}$ to be the relation on $G$ connecting $g, h$ if $g(n) \notin \operatorname{rng}(h\lceil m)$, and $h(n) \notin \operatorname{rng}(g\lceil m)$. Note that the relation $R_{n, m}$ is left invariant.

Let $B$ be the set of all finite injections $u$ from $\omega$ to $\omega$ such that $\operatorname{dom}(u) \in a$ and there is $g \in G$ with $u \subset g$. For $u \in B$, write $[u]=\{g \in G: u \subset g\}$.

Claim 5.2. Let $n<m$ be numbers in a and $u \in B$ is of size $n$. There are $g, h \in G$ which both extend $u$ and such that $g R_{n, m} h$ holds.

Proof. Since the relation $R_{n, m}$ is left invariant, it is enough to prove this for $u$ equal to the identity function on $n$. Let $g_{k}: k \in \omega$ be elements of $G$ which up to $n$ are equal to the identity, and $g_{k}(n)$ for $k \in \omega$ are pairwise distinct numbers. Thinning the collection if necessary, we may assume for every number $l \in[n, m)$ the numbers $g_{k}(l)$ are either pairwise distinct or all equal. Since the $g_{k}$ 's are injections, this means that for each $k_{0} \in \omega$ the set of all $k_{1}$ such that $g_{k_{0}}(n) \in \operatorname{rng}\left(g_{k_{1}}\lceil[n, m))\right.$, is finite. Thus, for every number $k$ large enough the pair $g=g_{0}$ and $h=g_{k}$ will work as required.

Let $A \subset B$ be a dense set such that

- the ranges of injections in $A$ are linearly ordered by inclusion;

- whenever $u \in B$ has no initial segment in $A$ then for every $m$ it has an extension $v \in B$ with no initial segment in $A$ and such that $m \in \operatorname{rng}(v)$.

It is easy to construct such a set $A$ by a bookkeeping argument. Let $C=G \backslash \bigcup_{k}\left[s_{k}\right]$; this is a closed nowhere dense subset of $C$. Note that if $u \in B$ then $C \cap[u]=0 \leftrightarrow \exists s \in A, s \subseteq u$. We claim this set $C$ works.

Suppose that $b \subset a$ is an infinite set. We must find group elements $g_{n} \in G$ for $n \in b$ such that for every $g \in S_{\infty} g \cdot \bigcup_{n}\left[g_{n}\lceil n] \cap C\right.$ is dense in $C$. Just use the claim to find the group elements so that for every $u \in B$ of length some $k \in a$ there are numbers $n_{0}<n_{1} \in b$ such that both $g_{n_{0}}, g_{n_{1}}$ extend $u$ and $g_{n_{0}} R_{k, n_{1}} g_{n_{1}}$ holds. We claim that $\left\{g_{n}: n \in b\right\}$ works.

Suppose that $g \in G$ is an element and $v \in B$ is such that $C \cap[v] \neq 0$. Let $k=\operatorname{dom}(v)$ and $u=g^{-1} \cdot v$, and find numbers $n_{0}<n_{1}$ in $b$ such that $g_{n_{0}}, g_{n_{1}}$ extend $u$ and are $R_{k, n_{1}}$-related. We claim that either $\left[g \cdot g_{n_{0}}\left\lceil n_{0}\right]\right.$ 
or $\left[g \cdot g_{n_{1}}\left\lceil n_{1}\right]\right.$ must have nonempty intersection with $C$ - this will suffice as both of these sets are subsets of $[v]$. If this is not the case, there must be injections $s_{0}, s_{1} \in A$ such that $s_{0} \subseteq g \cdot g_{n_{0}}\left\lceil n_{0}\right.$ and $s_{1} \subseteq g \cdot g_{n_{1}} \uparrow n_{1}$. However, neither $s_{0}, s_{1}$ can be a subset of $v$ since $C \cap[v] \neq 0$, so their respective ranges must contain the numbers $g \cdot g_{n_{0}}(k)$ and $g \cdot g_{n_{1}}(k)$ which do not belong to the range of the other sequence. This contradicts the assumption that the ranges of $s_{0}, s_{1}$ are linearly ordered by inclusion.

\section{REFERENCES}

[1] T. J. Carlson, Strong measure zero and strongly meager sets, Proc. Amer. Math. Soc. 118 (1993), 577-586. MR 1139474

[2] D. H. Fremlin, Measure theory, vol. 5, Set-theoretic measure theory, 2008; available at http://www.essex.ac.uk/maths/people/fremlin/mt5.2008/mt5.2008.tar.gz. MR 2462280

[3] F. Galvin, J. Mycielski and R. Solovay, Strong measure zero sets, Notices Amer. Math. Soc. 26: (1979), A-280.

[4] M. Hrušák, W. Wohofsky and O. Zindulka, Strong measure zero in separable metric spaces and Polish groups, Arch. Math. Logic 55 (2016), 105-131. MR 3453581

[5] T. Jech, Set theory, Academic Press, San Diego, 1978. MR 0506523

[6] M. Kysiak, On Erdös-Sierpiński duality between Lebesgue measure and Baire category, Ph.D. thesis, Warsaw University, 2000.

[7] P. B. Larson, The stationary tower forcing, University Lecture Series, vol. 32, American Mathematical Society, Providence, RI, 2004. Notes from Woodin's lectures. MR 2069032

[8] R. Laver, On the consistency of Borel's conjecture, Acta Math. 137 (1987), 151-169. MR 0422027

Michael Hrušák, Instituto de Matemáticas, Universidad Nacional Autónoma de México, Morelia, C. P. 58089, Michoacán, Mexico

Jindřich Zapletal, University of Florida, Gainesville, FL 32611, USA

E-mail address: zapletal@ufl.edu 